Article

\title{
Overcome Double Trouble: Baloxavir Marboxil Suppresses Influenza Thereby Mitigating Secondary Invasive Pulmonary Aspergillosis
}

\author{
Laura Seldeslachts $^{1}{ }^{(\mathbb{D}}$, Cato Jacobs $^{2}$, Birger Tielemans ${ }^{1}{ }^{(D)}$, Eliane Vanhoffelen ${ }^{1}$, Lauren Van der Sloten ${ }^{2}$, \\ Stephanie Humblet-Baron ${ }^{3}$, Lieve Naesens ${ }^{4} \mathbb{D}$, Katrien Lagrou ${ }^{5} \mathbb{D}$, Erik Verbeken ${ }^{6}$, Joost Wauters ${ }^{2}$ \\ and Greetje Vande Velde ${ }^{1, *(D)}$
}

1 Biomedical MRI Unit/MoSAIC, Department of Imaging and Pathology, KU Leuven, 3000 Leuven, Belgium; laura.seldeslachts@kuleuven.be (L.S.); birger.tielemans@kuleuven.be (B.T.); eliane.vanhoffelen@kuleuven.be (E.V.)

2 Laboratory for Clinical Infectious and Inflammatory Disorders, Department of Microbiology, Immunology and Transplantation, KU Leuven, 3000 Leuven, Belgium; cato.1.jacobs@uzleuven.be (C.J.); lauren.vandersloten@uzleuven.be (L.V.d.S.); Joost.wauters@uzleuven.be (J.W.)

3 Laboratory of Adaptive Immunity, Department of Microbiology, Immunology and Transplantation, KU Leuven, 3000 Leuven, Belgium; stephanie.humbletbaron@kuleuven.be

4 Laboratory of Virology and Chemotherapy, Rega Institute, Department of Microbiology, Immunology and Transplantation, KU Leuven, 3000 Leuven, Belgium; lieve.naesens@kuleuven.be

5 Laboratory of Clinical Bacteriology and Mycology, Department of Microbiology, Immunology and Transplantation, KU Leuven, 3000 Leuven, Belgium; katrien.lagrou@uzleuven.be

Citation: Seldeslachts, L.; Jacobs, C.; Tielemans, B.; Vanhoffelen, E.; Van der Sloten, L.; Humblet-Baron, S.; Naesens, L.; Lagrou, K.; Verbeken, E.; Wauters, J.; et al. Overcome Double Trouble: Baloxavir Marboxil Suppresses Influenza Thereby Mitigating Secondary Invasive Pulmonary Aspergillosis. J. Fungi 2022, 8, 1. https://doi.org/10.3390/ jof 8010001

Academic Editor: David S. Perlin

Received: 26 November 2021

Accepted: 17 December 2021

Published: 21 December 2021

Publisher's Note: MDPI stays neutral with regard to jurisdictional claims in published maps and institutional affiliations.

Copyright: (C) 2021 by the authors. Licensee MDPI, Basel, Switzerland. This article is an open access article distributed under the terms and conditions of the Creative Commons Attribution (CC BY) license (https:// creativecommons.org/licenses/by/ $4.0 /)$.
6 Department of Imaging and Pathology, KU Leuven, 3000 Leuven, Belgium; erik.verbeken@kuleuven.be

* Correspondence: greetje.vandevelde@kuleuven.be; Tel.: +32-16-33-09-24
Abstract: Influenza-associated pulmonary aspergillosis (IAPA) is a global recognized superinfection in critically ill influenza patients. Baloxavir marboxil, a cap-dependent endonuclease inhibitor, is a newly approved anti-influenza therapeutic. Although the benefits as a treatment for influenza are clear, its efficacy against an influenza- $A$. fumigatus co-infection has yet to be determined. We investigated the therapeutic effect of baloxavir marboxil in a murine model for IAPA. Immunocompetent mice received intranasal instillation of influenza A followed by orotracheal inoculation with Aspergillus fumigatus 4 days later. Administration of baloxavir marboxil or sham was started at day 0 , day 2 or day 4 . Mice were monitored daily for overall health status, lung pathology with micro-computed tomography $(\mu \mathrm{CT})$ and fungal burden with bioluminescence imaging (BLI). In vivo imaging was supplemented with virological, mycological and biochemical endpoint investigations. We observed an improved body weight, survival and viral clearance in baloxavir marboxil treated mice. $\mu \mathrm{CT}$ showed less pulmonary lesions and bronchial dilation after influenza and after Aspergillus co-infection in a treatment-dependent pattern. Furthermore, baloxavir marboxil was associated with effective inhibition of fungal invasion. Hence, our results provide evidence that baloxavir marboxil mitigates severe influenza thereby decreasing the susceptibility to a lethal invasive Aspergillus superinfection.

Keywords: influenza-associated pulmonary aspergillosis (IAPA); baloxavir marboxil; multimodal preclinical imaging-supported mouse model; Aspergillus fumigatus; influenza

\section{Introduction}

Over the past four decades, viral associated pulmonary aspergillosis (VAPA), such as influenza-associated pulmonary aspergillosis (IAPA) or more recently COVID-19-associated pulmonary aspergillosis (CAPA), have become new entities in the world as serious lifethreatening infectious diseases [1-10]. The lethal combination of influenza and Aspergillus leads to a poor outcome characterized by a longer ICU stay and an almost doubled mortality rate in comparison with severe influenza patients $[4,5,11]$. Understanding the synergism 
between the virus and the fungus is of utmost importance. We recently developed a preclinical animal model of IAPA disease, confirming influenza as a strong risk factor for developing invasive pulmonary aspergillosis (IPA) in immunocompetent mice [12,13].

Ever since the flu pandemic of 2009, the neuraminidase inhibitor (NAI) oseltamivir has been used as standard-of-care treatment to suppress influenza. This drug prevents the release and spread of the influenza virus from the host cell by inhibiting the viral neuraminidase. We found that early oseltamivir modulated the severity of influenza thereby mitigating the susceptibility to IAPA in an immunocompetent murine model [12]. In addition, in the clinical setting, prophylactic oseltamivir proved to be effective in prevention of IAPA in children after haploidentical hematopoietic cell transplantation [14]. However, despite the beneficial effects of oseltamivir, we need to have alternative antivirals at hand. Indeed, it is hypothesized that oseltamivir may play a harmful role in the host immune defense against fungal superinfection. Human and mice express neuraminidases (NEU1NEU4), which show similar tertiary structures and active-site amino acids as the viral sialidases. NAIs like oseltamivir, might therefore inhibit the endogenous neuraminidase activity on the host immune cells and modulate host-pathogen interaction $[15,16]$. Although only limited inhibitory effects of oseltamivir on mammalian sialidases were reported [15], Dewi et al. (2021) showed that oseltamivir influences the host response against $A$. fumigatus in vitro by inhibiting the endogenous neuraminidases resulting in an impaired capacity of human peripheral blood mononuclear cells (PBMCs) and murine splenocytes to kill Aspergillus [16]. Furthermore, in vivo, an increased susceptibility to IPA with higher fungal burden and mortality was found [16].

In addition to a possible effect of blocking the host neuraminidase by NAI, emergence of NAI resistant influenza strains can occur that warrant the need for alternative antivirals. Currently, resistance to NAI in community isolates remains at low levels $(<1 \%)$, however higher incidences of oseltamivir-resistant variants exist [17,18]. In 2009, the influenza A (H1N1)pdm09 virus with the NA-H275Y amino acid substitution occurred as a widespread oseltamivir-resistant influenza strain [17,19-21]. In a French multicenter observational cohort study, a highly reduced sensitivity to oseltamivir was found in $23 \%$ of the patients with a severe influenza A(H1N1)pdm09 [NA-H275Y] pneumonia [19]. If resistance occurs, it will be important to use other effective antiviral treatments which can lower the severity of the influenza infection and thereby mitigate the susceptibility to IPA [12]. Resistance together with the possible effect of blocking the host neuraminidase indicate the importance to preclinically assess new antiviral drugs, relying on different modes of actions, on the effectiveness in IAPA.

Baloxavir marboxil, a cap-dependent endonuclease inhibitor, is a novel anti-influenza therapeutic approved by Japan and the U.S. Food and Drug Administration (FDA) in 2018 and the European Medicine Agency (EMA) in 2021 [22,23]. Different clinical trials have shown the superior effect of a single-dose baloxavir marboxil in alleviating influenza symptoms compared to placebo and reducing viral load compared to oseltamivir and placebo $[24,25]$. Additionally in preclinical studies, oral administration of baloxavir marboxil (15 mg/ $\mathrm{kg}$, twice daily) was more efficient than oseltamivir in reducing the mortality rate, body weight loss and viral titers, even with a delay in treatment initiation of 4 or 5 days post-influenza infection in mice [26,27]. Although the benefits of baloxavir marboxil as a treatment method for influenza are well described, the effectiveness of the antiviral drug against an influenza- $A$. fumigatus co-infection has yet to be determined.

Hence, we investigated the time-dependent treatment effect of baloxavir marboxil in suppressing influenza and its efficacy to prevent a secondary invasive pulmonary aspergillosis in an imaging-based immunocompetent murine model of IAPA. 


\section{Materials and Methods}

\subsection{Influenza Virus and Aspergillus Strain}

A mouse-adapted influenza virus A/H3N2/Ishikawa/7/82 and a red-shifted thermostable firefly luciferase $A$. fumigatus strain were prepared, as previously described [12] (manuscript in consideration [28]).

\subsection{Mouse Model}

All animal experiments were approved by the KU Leuven Ethical Committee for animal research (license p074/2018). Mice were kept in individually ventilated cages with free access to food and water in a conventional animal facility. An antibiotic $(50 \mathrm{mg} / \mathrm{kg} /$ day, Baytril $^{\circledR}$, Bayer, Leverkusen, Germany) was added to the drinking water to prevent bacterial infections. Eight- to ten-week-old immunocompetent male C57BL/6NTac mice (internal stock of the Laboratory Animal Centre, KU Leuven, Leuven, Belgium) were randomly assigned to experimental groups with a sample size of 5 per group per experiment. The experiment was repeated twice. The mouse model was developed as previously described [12]. Briefly, mice received intranasal instillation of 10 plaque forming units (PFU) influenza virus on day $0(10 \mu \mathrm{L}$ per nostril $)$ and orotracheally inoculation with $20 \mu \mathrm{L}$ of $A$. fumigatus spore solution $\left(5 \times 10^{9}\right.$ conidia $\left./ \mathrm{mL}\right)$ under inhalation anesthesia with isoflurane (1.5-2\% in 100\% oxygen, Primal Critical Care) on day 4. Baloxavir marboxil $(15 \mathrm{mg} / \mathrm{kg}$ ) or phosphate-buffered saline (PBS) treatment was administered twice daily by oral gavage (Figure 1A). The dose of $15 \mathrm{mg} / \mathrm{kg}$ twice daily mimic the plasma concentration of $6.85 \mathrm{ng} / \mathrm{mL}$ in humans and therefore used to predict clinical effectiveness [26,27]. During the experiments, mice were longitudinally monitored for body weight and non-invasively scanned with micro-computed tomography ( $n=10$ per group) and bioluminescence imaging ( $n=5$ per group). Mice were anesthetized by inhalation of $1.5-2 \%$ isoflurane in $100 \%$ oxygen during scanning. Sacrifice was performed at a predefined endpoint on day 7, with an overdose of pentobarbital via intraperitoneal injection. Bronchoalveolar lavage (BAL) fluid, right and left lung lobes were collected as previously described [12]. Left and right lungs were used for respectively histopathology ( $n=5$ per group) and quantification of virus titers and fungal burden ( $n=10$ per group).

\subsection{Micro-Computed Tomography ( $\mu \mathrm{CT})$}

Acquisition, visualization and quantification of $\mu \mathrm{CT}$ were performed as previously described [12]. Briefly, for $\mu \mathrm{CT}$, a SkyScan 1278 (Bruker $\mu \mathrm{CT}$, Kontich, Belgium) was used for acquisition with the following scan parameters ( $50 \mathrm{kVp}$ X-ray source, $1 \mathrm{~mm}$ aluminum $\mathrm{X}$-ray filter, $350 \mu \mathrm{A}$ current, $150 \mathrm{~ms}$ exposure time per projection, $0.9^{\circ}$ increments over a total angle of $220^{\circ}$ ). Visualization of reconstructed $\mu \mathrm{CT}$ were performed with DataViewer, CTan and CTvox. Semi-quantitative scoring was performed by two blinded researchers according to Seldeslachts et al. (2021) [12]. For quantification, imaging-derived biomarkers of lung pathology (non-aerated lung volume (NALV)) was determined based on manually delineation of region of interest (ROI) resulting in a volume of interest (VOI) covering the entire lung and the previously determined threshold of 108-255 greyscale. Bronchial dilation was determined by manually delineation of ROI of the airways and a threshold of 0-90 greyscale.

\subsection{Bioluminescence Imaging (BLI)}

For in vivo and ex vivo BLI, data acquisition, visualization and quantification were performed as previously described [12]. Briefly, the IVIS Spectrum System (Perkin-Elmer, Hopkinton, MA, USA) was used with following settings (exposure time 1 min, medium binning, F/stop of 1 and subject height of $1.5 \mathrm{~cm}$ in vivo and $1 \mathrm{~cm}$ for ex vivo). Quantification of BLI data was performed with Living Image Software version 4.7.3 as previously described [12]. The highest total photon flux from consecutive acquisitions was used for further analysis. 
A.

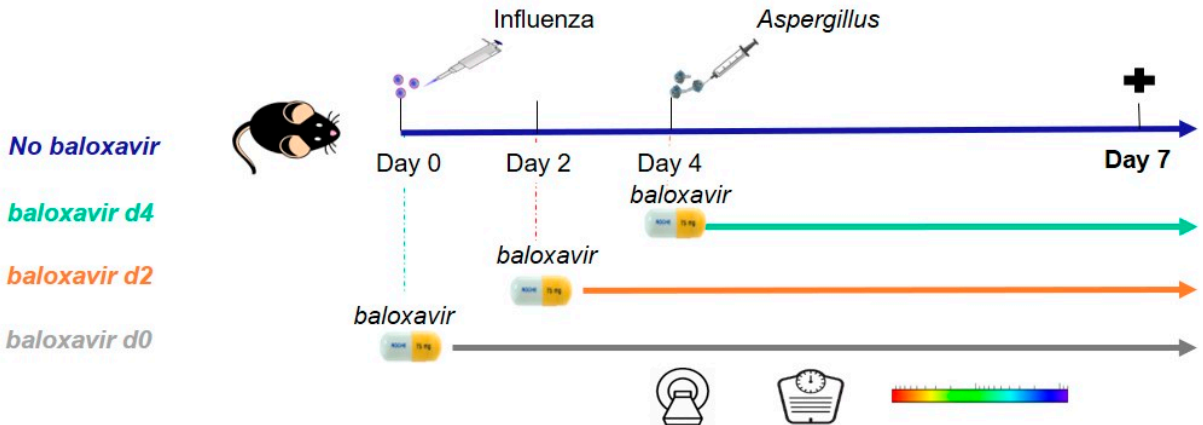

B.

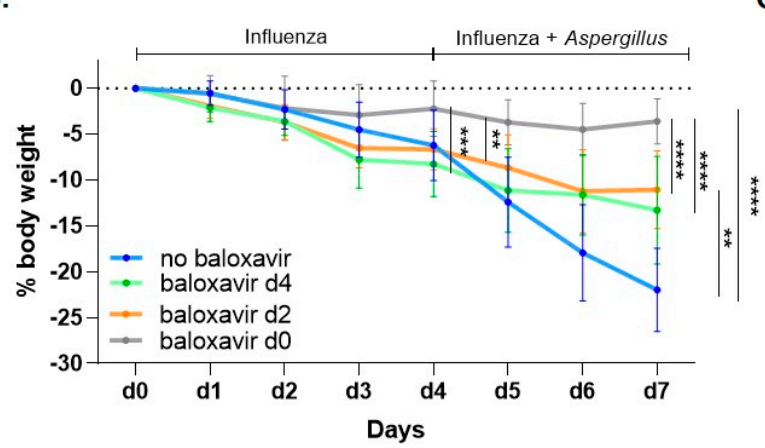

C.

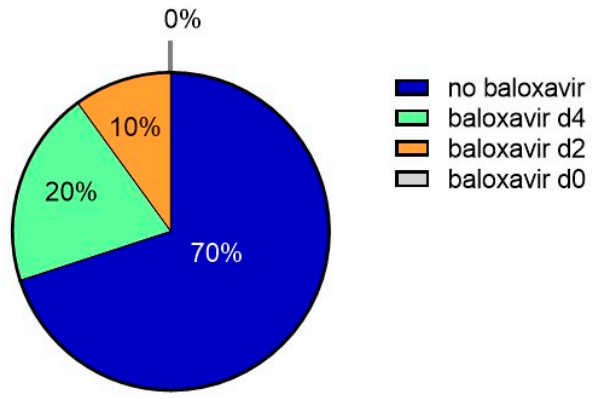

Figure 1. Baloxavir marboxil treatment improves survival and body weight loss. (A) Experimental setup: immunocompetent mice received intranasal instillation influenza A followed by orotracheal inoculation with Aspergillus fumigatus 4 days later. Baloxavir marboxil treatment or sham (no baloxavir, blue) was started at day 4 (green), day 2 (orange) or day 0 (grey) up to day 7. Mice were longitudinally monitored for body weight, with micro-computed tomography $(\mu \mathrm{CT})$ and bioluminescence imaging (BLI). (B) Relative body weight evolution of influenza/Aspergillus co-infected mice receiving sham treatment (no baloxavir, blue, $n=10$ mice), baloxavir marboxil treatment starting from day 4 (green, $n=10$ mice), baloxavir marboxil treatment started on day 2 (orange, $n=10$ mice) and baloxavir marboxil treatment from day 0 (grey, $n=10$ mice). (C) Pie chart representing survival expressed as the percentage (\%) of animals reaching humane endpoint ( $>20 \%$ body weight loss) at day 7 . Differences were considered significant if $p$ was smaller or equal to 0.05 . ${ }^{* *} p<0.01,{ }^{* * *} p<0.001$, $^{* * * *} p<0.0001$. Data are represented as mean $\pm \mathrm{SD}$.

\subsection{Quantification of Virus and Fungal Titers}

Viral and fungal load detection was similar as Seldeslachts et al. (2021) [12]. Briefly, for fungal load detection by colony-forming unit (CFU) counting, serial dilutions of right lung homogenates were plated on Sabouraud agar plates and incubated at $37^{\circ} \mathrm{C}$ for $48 \mathrm{~h}$. Reported CFU counts represent average values of duplicate plating of each dilution. Viral titer determination was performed by adding a serial dilutions of right lung homogenate supernatants to Madin-Darby Canine Kidney cells (MDCK) in quadruplicate, incubation at $35^{\circ} \mathrm{C}$ during $72 \mathrm{~h}$ and assessment of a cytopathic effect by microscopy scoring.

\subsection{Histopathology}

Each left lung lobe ( $n=5$ per group) was fixed with $10 \%$-formalin, 24 h post fixated and then stored in PBS- $0.1 \%$ sodium-azide at $4{ }^{\circ} \mathrm{C}$. Subsequently, samples were paraffin embedded and $5 \mu \mathrm{M}$ sagittal sections were stained with hematoxylin-eosin (H\&E) and Grocott's methenamine silver staining (GMS). Histological tissue sections were scored by a blinded pathologist (EVB).

\subsection{Statistical Analysis}

Statistics were performed using GraphPad Prism (version 8.1.2, GraphPad Software, San Diego, CA, USA). Longitudinal data were analyzed with two-way ANOVA with 
Tukey's multiple comparison test (repeated measurements performed from day 0-day 4 and day 4-day 7) and endpoint data with one-way ANOVA with Tukey's multiple comparisons test or Kruskal-Wallis test. All graphs represent mean values \pm SD. Differences were considered significant if $p$ was smaller or equal to 0.05 . ${ }^{*} p<0.05,{ }^{* *} p<0.01,{ }^{* * *} p<0.001$, $* * * * \quad p<0.0001$. $n$ values represent the number of animals.

\section{Results}

\subsection{Baloxavir Marboxil Treatment Improves Survival and Limits Body Weight Loss}

Recently, we identified influenza as a strong risk factor for developing invasive pulmonary aspergillosis (IPA) in immunocompetent mice [12]. To assess the time-dependent treatment effect of baloxavir marboxil on the severity of influenza and subsequent susceptibility to IPA in this double-hit mouse model, we treated mice with baloxavir marboxil or PBS started at day 0, day 2 or day 4 up to day 7 (Figure 1A). During the influenza infection (i.e., day 0-day 4), we observed markedly less body weight loss in the baloxavir marboxil prophylactic treated group in comparison with other groups (Figure 1B). Upon Aspergillus co-infection (i.e., day 4-day 7), the non-treated mice showed a faster and more substantial body weight loss (Figure 1B) as well as a fatal clinical deterioration in $70 \%$ of the animals by day 7 , the predetermined endpoint of this study (Figure 1C). In sharp contrast, all baloxavir marboxil-treated mice experienced only a moderate body weight loss (Figure 1B) and less animals reached the humane endpoint at day 7 (Figure 1C) the earlier treatment was started. Hence, baloxavir marboxil limits body weight loss and improves survival in a treatment-onset dependent manner.

\subsection{Baloxavir Marboxil Reduces Infiltrates and Bronchial Dilation on Lung $\mu \mathrm{CT}$ after Influenza and Aspergillus Co-Infection}

To further investigate the impact of baloxavir marboxil on the lung lesion development during influenza pneumonia and upon Aspergillus superinfection, we longitudinally followed up the mice through non-invasive $\mu \mathrm{CT}$. Viral induced lung pathology and bronchial dilation (day 0-day 4) were significantly reduced in prophylactic treated mice in comparison with other groups, coinciding with only a moderate body weight loss (Figure 2A,B,E). During Aspergillus co-infection (day 4-day 7), visual assessment of 3D rendered aerated lung volumes showed severe diffuse pulmonary consolidations of entire lung lobes and bronchial dilation in non-treated mice while baloxavir marboxil treatment resulted in less to no pulmonary infiltrates or bronchial dilation the earlier treatment was started (Figure 2A,B,E). In line with the visual observations, the quantification of longitudinal $\mu \mathrm{CT}$-derived biomarkers (non-aerated lung volume (NALV) and bronchial dilation) confirmed our findings of a time-dependent reduction in viral and viral-fungal pulmonary lesion development, as well as bronchial dilation during baloxavir marboxil treatment (Figure 2C,D,F). Together, these results show that baloxavir marboxil limits lung pathology on $\mu \mathrm{CT}$ after influenza and after Aspergillus co-infection.
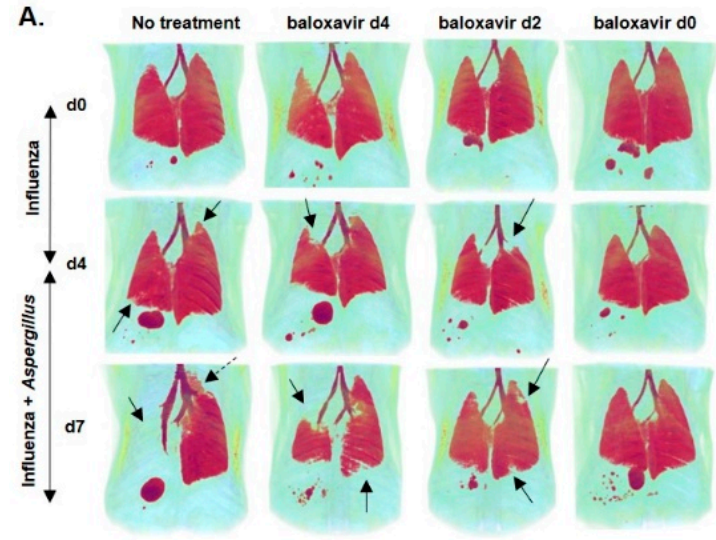

B.

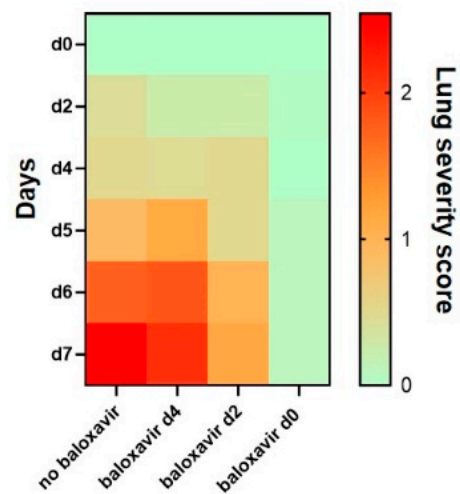

Figure 2. Cont. 
C.

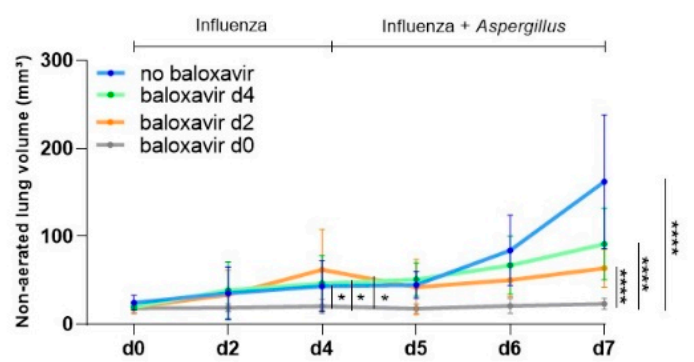

E.

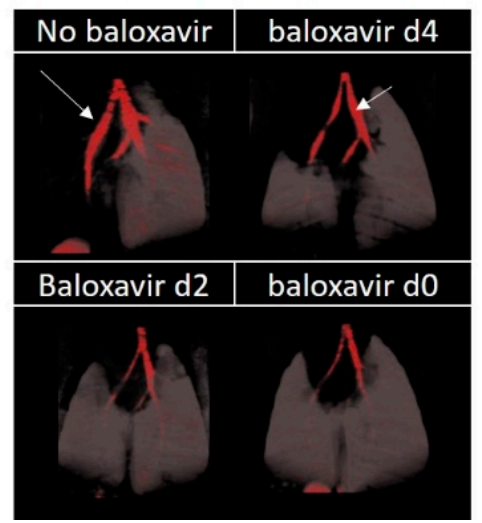

F.
D.

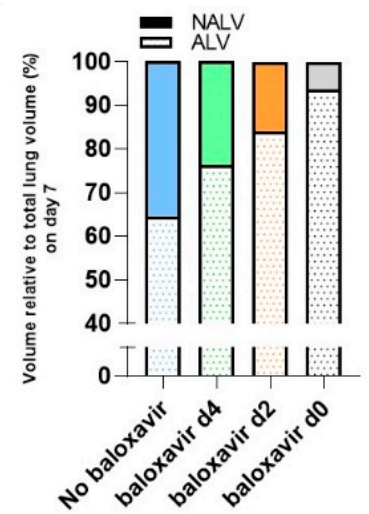

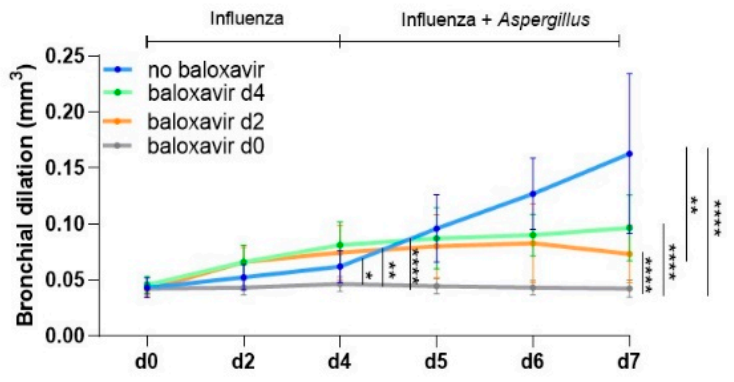

Figure 2. Baloxavir marboxil treatment reduces viral and viral-fungal pulmonary pathology and bronchial dilation. (A) Longitudinal three-dimensional (3D) visualization of aerated lung volume acquired with $\mu \mathrm{CT}$. Red: aerated lung tissue, Black arrows point at pulmonary infiltrates/lung pathology and dashed arrow to bronchial dilation. (B) Heatmap representation of lung severity scores based on transverse lungs $\mu \mathrm{CT}$ images of non-treated (sham, no baloxavir), baloxavir marboxil from day 4, baloxavir marboxil from day 2 and baloxavir marboxil from day 0 treated mice. Red: severe pulmonary lesions, orange: moderate pulmonary lesions, green: no pulmonary lesions. (C) Graph represents longitudinal $\mu \mathrm{CT}$-derived biomarker "non-aerated lung volume" (pulmonary lesions) of immunocompetent influenza/Aspergillus co-infected mice that received no baloxavir (sham, blue, $n=10$ mice), baloxavir marboxil treatment-onset from day 4 (green, $n=10$ mice), baloxavir marboxil treatment-onset from day 2 (orange, $n=10$ mice) and baloxavir marboxil treatmentonset from day 0 (grey, $n=10$ mice). (D) $\mu$ CT-derived biomarkers: non-aerated lung volume and aerated lung volume relative to total lung volume on day 7 of non-treated (sham, no baloxavir, blue, $n=10$ mice), baloxavir marboxil from day 4 (green, $n=10$ mice), baloxavir marboxil from day 2 (orange, $n=10$ mice) and baloxavir marboxil from day 0 (grey, $n=10$ mice). (E) Longitudinal 3D visualization of aerated lung volume acquired with $\mu \mathrm{CT}$. Grey: aerated lung tissue, Red: trachea with arrow bronchial dilation. (F) Quantification of bronchial dilation determined by manually delineation of the region of interest (ROI) of the airways of mice receiving no baloxavir marboxil (sham, blue, $n=10$ mice), baloxavir marboxil treatment-onset from day 4 (green, $n=10$ mice), baloxavir marboxil treatment-onset from day 2 (orange, $n=10$ mice) and baloxavir marboxil treatment-onset from day 0 (grey, $n=10$ mice). Statistical analysis for longitudinal graph: two-way ANOVA with Tukey's multiple comparisons test repeated measurements performed from day 0 until day 4 (influenza infection) and day 4 until day 7 (influenza-Aspergillus co-infection). Differences were considered significant if $p$ was smaller or equal to $0.05 .{ }^{*} p<0.05,{ }^{* *} p<0.01{ }^{* * * *} p<0.0001 . n$ values represent the number of animals. Data are represented as mean \pm SD. 


\subsection{Baloxavir Marboxil Effectively Clears Influenza}

Next, we determined the viral load in lung tissue at endpoint in treated and nontreated mice (Figure 3). The viral load at day 7 was high in untreated group, while in the baloxavir marboxil treated groups the viral load was significantly reduced regardless of the of the timing of baloxavir marboxil administration. So baloxavir marboxil has an effective viricidal activity against influenza.

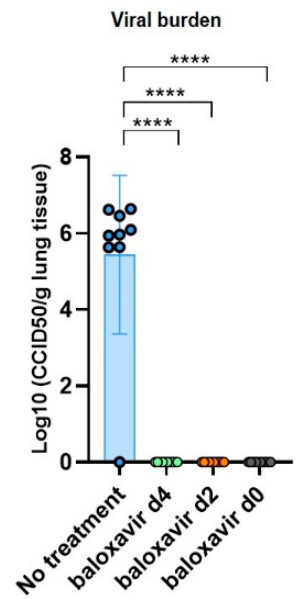

Figure 3. Baloxavir marboxil has an effective viricidal activity against influenza. Viral burden: graph representing the $\log _{10}$ CCID50 per gram lung tissue receiving no baloxavir marboxil (sham, blue, $n=10$ mice), baloxavir marboxil treatment-onset from day 4 (green, $n=10$ mice), baloxavir treatmentonset from day 2 (orange, $n=10$ mice) and baloxavir marboxil treatment-onset from day 0 (grey, $n=10$ mice). Differences were considered significant if $p$ was smaller or equal to $0.05 .{ }^{* * * *} p<0.0001$. Data are represented as individual data-points, mean \pm SD.

\subsection{Baloxavir Marboxil Reduces the Risk for a Secondary Invasive Pulmonary Aspergillosis}

Having established fewer lung pathology and viral clearance after baloxavir marboxil treatment, we next explored the effectiveness in inhibiting invasive fungal disease development through longitudinal BLI of luciferase-tagged A. fumigatus as a proxy of fungal burden and CFU counts. In vivo pulmonary bioluminescence signal intensity was significantly reduced in all baloxavir marboxil treated mice compared with non-treated mice towards day 7 (Figure 4A,B). Furthermore, the fungal load in lung homogenates of prophylactic treated mice was significantly lower than in non-treated mice, measured by ex vivo BLI (close to background signal) and CFU (Figure 4C,D). Strikingly, A. fumigatus was detected in all groups. Histopathology confirmed the presence of $A$. fumigatus conidia in all groups but identified a clear morphological/phenotypical difference (Figure 4E). In baloxavir marboxil treated mice no airway invasive hyphae were detected while clearly present in non-treated mice (Figure 4E). These data suggest that baloxavir marboxil is effective in preventing a secondary invasive pulmonary aspergillosis.

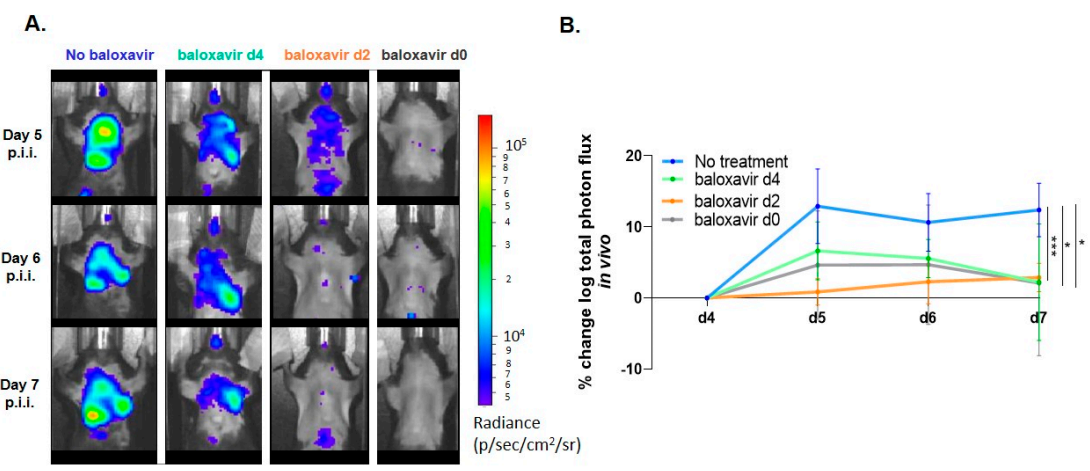

Figure 4. Cont. 
c.

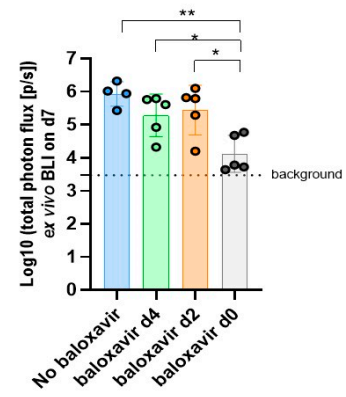

D.

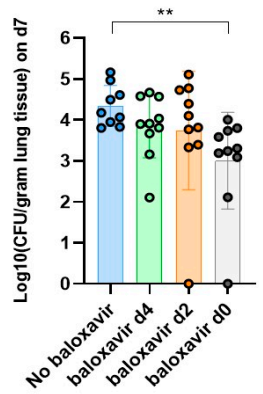

E.

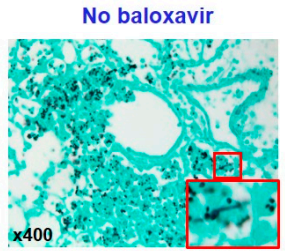

baloxavir d4

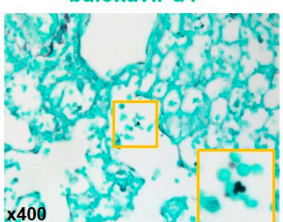

No baloxavir

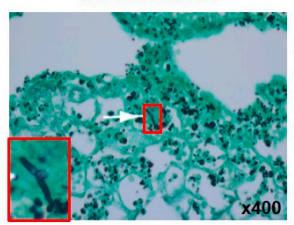

baloxavir d2

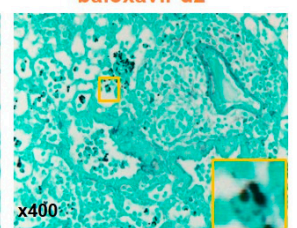

No baloxavir

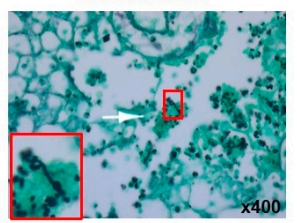

baloxavir do

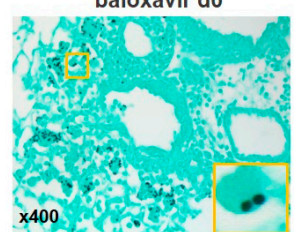

Figure 4. Baloxavir marboxil is effective in preventing secondary invasive pulmonary aspergillosis. (A) Bioluminescence images (BLI) of one mouse per group acquired on day 5, day 6 and day 7 post influenza-infection. (B) Quantification of total photon flux from in vivo BLI based on ROI covering the complete lung region and represented as the $\%$ change of the $\log _{10}$ (total photon flux) values in comparison with baseline of influenza-Aspergillus day 4 co-infected mice without baloxavir marboxil (blue, $n=5$ mice), baloxavir marboxil from day 4 (green, $n=5$ mice), baloxavir marboxil from day 2 (orange, $n=5$ mice) and baloxavir marboxil from day 0 (grey, $n=5$ mice). Longitudinal statistical analysis: repeated measurements two-way ANOVA with Tukey's multiple comparisons test performed from day 4 until day 7. (C) Quantification of the total photon flux from ex vivo BL images based on ROI covering the well containing undiluted lung homogenates on day 7 from no baloxavir marboxil (sham, blue, $n=5$ mice), baloxavir marboxil from day 4 (green, $n=5$ mice), baloxavir marboxil from day 2 (orange, $n=5$ mice) and baloxavir marboxil from day 0 (grey, $n=5$ mice) treated mice. (D) Graph representing the $\log _{10}$ CFU count per gram lung tissue on day 7 from non-treated (no baloxavir, blue, $n=10$ mice), baloxavir marboxil from day 4 (green, $n=10$ mice), baloxavir marboxil from day 2 (orange, $n=10$ mice) and baloxavir marboxil from day 0 (grey, $n=10$ mice). (E) Histological Grocott's methenamine silver staining (GMS) (magnification $\times 400$ ) of left lung of different groups (no baloxavir, baloxavir marboxil treatment from day 4 , baloxavir marboxil treatment from day 2, baloxavir marboxil treatment from day 0) sacrificed on day 7. Hyphae (red box), intracellular conidia (yellow box). Differences were considered significant if $p$ was smaller or equal to $0.05 .{ }^{*} p<0.05,{ }^{* *} p<0.01,{ }^{* * *} p<0.001$. Data are represented as mean $\pm \mathrm{SD}$.

\subsection{Baloxavir Marboxil Treatment Onset-Dependent Reduction in Lung Pathology and Local Immune Response}

We further zoomed in on the effectiveness of baloxavir marboxil in preventing severe lung pathology and extensive local immune response after influenza-Aspergillus coinfection. We found a clear morphological difference between treated and non-treated mice characterized by a treatment-onset dependent drastic decrease in distribution of affected lung tissue and less severe to no epithelial damage (erosion versus stimulation/cytotoxicity) (Figure 5A,B). Histological general inflammatory subset analysis of mono- versus polymorphonuclear leukocyte populations showed a marked decrease in neutrophils in treated mice compared to non-treated mice (Figure 5C,D). The same observation was found in 
bronchoalveolar lavage fluid (BALF) where baloxavir marboxil treatment resulted in a treatment-onset dependent decrease in neutrophil infiltration. In BALF this decrease in neutrophil infiltration was in favor of the mononuclear infiltrate macrophage population (Figure 6A,B). Collectively, these results show less severe epithelial damage, limited pneumonic extension and a reduced local immune response in baloxavir marboxil treated mice with a treatment-onset dependent pattern.

A.

\begin{tabular}{|c|c|c|c|c|}
\hline & \multicolumn{2}{|c|}{ Bronchovascular axis } & \multicolumn{2}{|c|}{ Alveolar parenchyma } \\
\hline & Distribution & Epithelium & Distribution & Epithelium \\
\hline & $\mathrm{D}$ & $E / S$ & $\mathrm{D}$ & I \\
\hline & D & $\mathrm{E} / \mathrm{S}$ & D & $E / S$ \\
\hline & MF & $\mathrm{E} / \mathrm{S}$ & MF & $\mathrm{E}$ \\
\hline & MF & $\mathrm{E} / \mathrm{S}$ & MF & $\mathrm{E}$ \\
\hline \multirow[t]{5}{*}{ No baloxavir } & D & $\mathrm{E} / \mathrm{S}$ & MF & $\mathrm{E}$ \\
\hline & $\bar{D}$ & $E / S$ & MF & $\bar{E}$ \\
\hline & MF & $\mathrm{E} / \mathrm{S}$ & $\mathrm{F}$ & $\mathrm{E}$ \\
\hline & D & $\mathrm{E} / \mathrm{S}$ & D & $\mathrm{E}$ \\
\hline & $\mathrm{F}$ & $s$ & $\mathrm{~F}$ & $\mathrm{E}$ \\
\hline \multirow[t]{5}{*}{ baloxavir d4 } & $\mathrm{F}$ & $\mathrm{s}$ & $\mathrm{F}$ & 1 \\
\hline & $\mathrm{F}$ & $\mathrm{s}$ & $\mathrm{F}$ & 1 \\
\hline & MF & $\mathrm{s}$ & MF & $\mathrm{E}$ \\
\hline & MF & s & MF & $\mathrm{E}$ \\
\hline & $\mathrm{F}$ & s & $\mathrm{F}$ & I \\
\hline \multirow[t]{5}{*}{ baloxavir $\mathrm{d} 2$} & MF & $E / S$ & MF & 1 \\
\hline & $\mathrm{F}$ & $s$ & $\mathrm{~F}$ & I \\
\hline & $\mathrm{F}$ & I & l & I \\
\hline & MF & $\mathrm{s}$ & $\mathrm{F}$ & $\mathrm{s}$ \\
\hline & $\mathrm{F}$ & $E / S$ & $\mathrm{~F}$ & $\mathrm{~s}$ \\
\hline baloxavir $\mathrm{d} 0$ & MF & $\mathrm{s}$ & MF & $\mathrm{s}$ \\
\hline
\end{tabular}

•Diffuse (D), multifocal (MF), focal (F)), $E=$ erosion, $S=$ stimulation (cytotoxicity)

c.

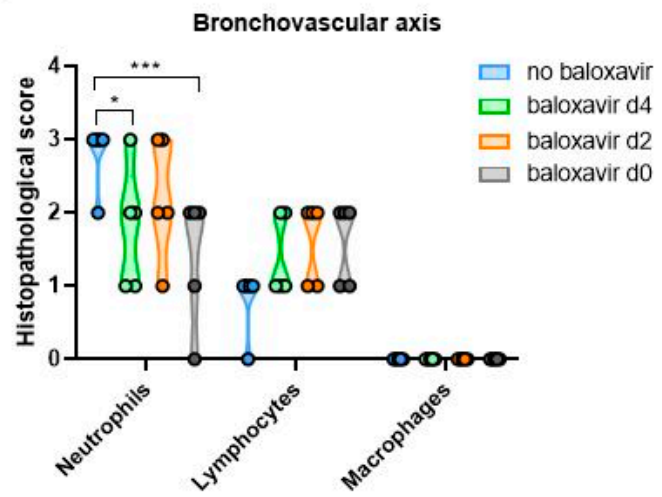

B.

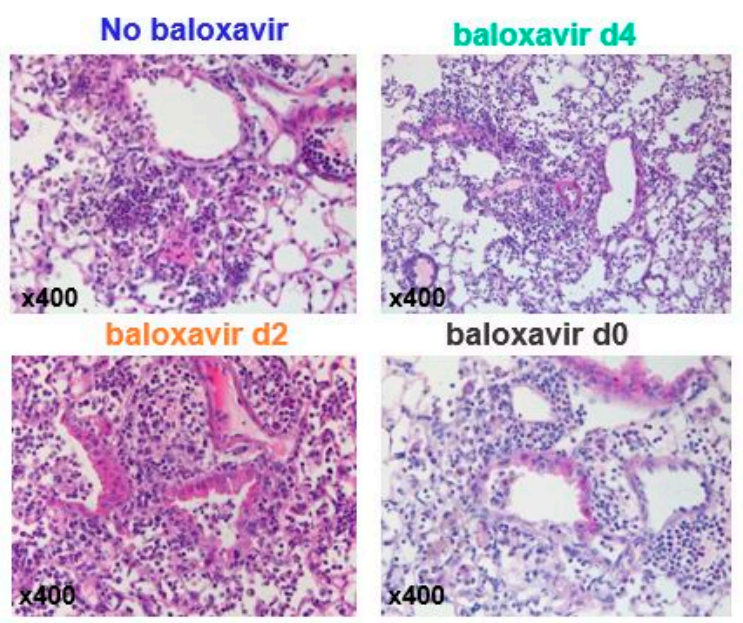

D.

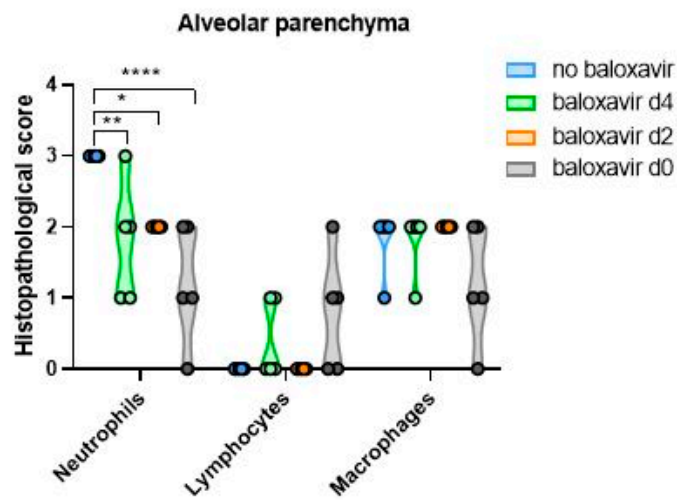

Figure 5. Baloxavir marboxil treatment onset-dependent reduction in lung pathology (A) Histopathological scores including the type of distribution (diffuse (D), multifocal (MF), focal (F)) for $n=5$ mice per group. (B) Histological hematoxylin and eosin $(\mathrm{H} \& \mathrm{E})$ staining (magnification $\times 400$ ) of no baloxavir marboxil, baloxavir marboxil from day 4 , baloxavir marboxil from day 2 and baloxavir marboxil from day 0. (C,D) Histological general inflammatory subset analysis (neutrophils, macrophages, lymphocytes) in bronchovascular axis (C) and alveolar parenchyma (D) from non-treated (no baloxavir, blue, $n=5$ mice), baloxavir marboxil from day 4 (green, $n=5$ mice), baloxavir marboxil from day 2 (orange, $n=5$ mice) and baloxavir marboxil from d0 (grey, $n=5$ mice). Differences were considered significant if $p$ was smaller or equal to $0.05 .{ }^{*} p<0.05,{ }^{* *} p<0.01,{ }^{* * *} p<0.001,{ }^{* * * *} p<0.0001$. Data are represented as mean $\pm \mathrm{SD}$. 
A.

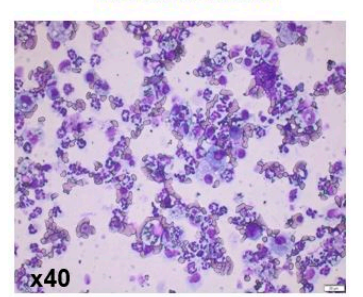

baloxavir d2

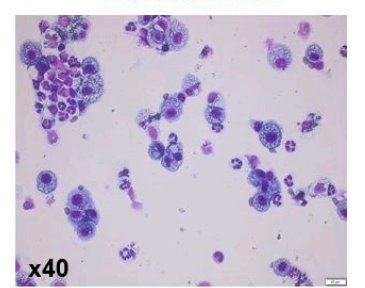

baloxavir d4

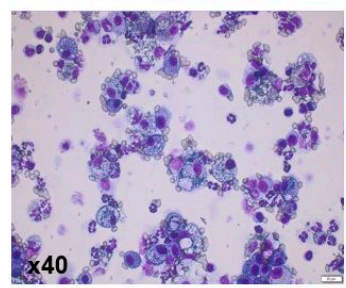

baloxavir do

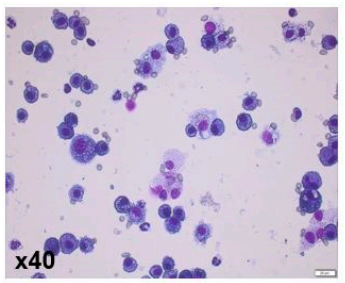

B.

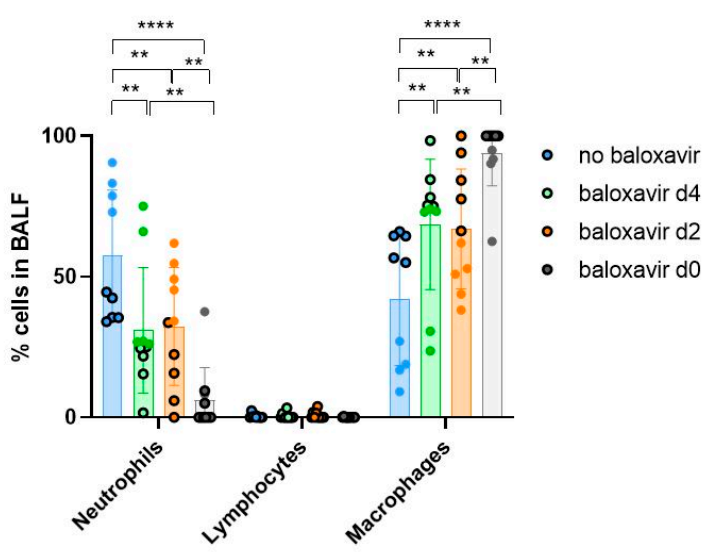

Figure 6. Treatment onset-dependent reduction in local immune response. (A,B) Visualization (A) and quantification (B) of mono- and polymorphonuclear leukocyte populations in bronchoalveolar lavage fluid from non-treated (no baloxavir, blue, $n=10$ mice), baloxavir marboxil from day 4 (green, $n=10$ mice), baloxavir marboxil from day 2 (orange, $n=10$ mice) and baloxavir marboxil from day 0 (grey, $n=10$ mice). Color fill vs. black contour color filled dot represents results from two different experiments. ${ }^{* *} p<0.01,{ }^{* * * *} p<0.0001$. Graphs are represented individual data points, mean \pm SD.

\section{Discussion}

Here we report the therapeutic effectiveness of baloxavir marboxil in suppressing influenza and thereby preventing a secondary invasive pulmonary aspergillosis in a nonimmunocompromised host. We herewith corroborate influenza as an independent risk factor for developing IPA.

The antiviral drug, baloxavir marboxil, is known to show therapeutic activity in uncomplicated influenza patients and preclinical models of influenza A and B virus infections, including resistant strains [24]. On top of this, we now preclinically show that baloxavir marboxil is effective during an influenza- $A$. fumigatus superinfection. This is evidenced by firstly an improved survival, ameliorated body weight and suppressed replication of influenza virus in the lungs even when treatment with baloxavir marboxil was delayed. Several preclinical and clinical studies in a mono-infection setting have already shown a time-dependent reduction in lung viral titers, decreased mortality and improved body weight after baloxavir marboxil treatment [24-27]. We now demonstrated that this is also the case after fungal co-infection in a preclinical mouse model of IAPA. Secondly, we longitudinally visualized for the first time a baloxavir marboxil treatment-onset dependent reduction in pulmonary lesions and bronchial dilation after influenza and after Aspergillus co-infection. Thirdly, limited influenza severity in mice treated with baloxavir marboxil was associated with reduced fungal burden development and morphological absence of airway invasive hyphae in the lungs. Finally, antiviral therapy reduced the excess infiltration of inflammatory neutrophils into the lung, thus protecting the lungs from severe pneumonic damage as well as an overreacting local immune response in bronchoalveolar lavage fluid. Additionally, baloxavir marboxil treatment was associated with higher levels of alveolar macrophages in BALF. This could point to a better anti-fungal lung macrophage defense and thus lower fungal burden $[29,30]$. The significant lower number of alveolar macrophages in non-treated mice could point to a potential immune dysregulation through which influenza predisposes to a fungal superinfection. It is known that type I IFN induced by influenza can inhibit the production of the chemokine CCL2, which is important for macrophage recruitment [31,32]. Besides an effect on recruitment, influenza virus infection can result in depletion of alveolar macrophages and can impair the macrophage phago- 
cytic activity important for fungal clearance [31-33]. However, further comprehensive immunological studies are needed to identify the immunological mechanism behind IAPA. Thus, baloxavir marboxil is effective in decreasing susceptibility to influenza-associated pulmonary aspergillosis in a murine model even when treatment was delayed. We herewith preclinically show that baloxavir marboxil forms a good alternative antiviral drug which can be used in case oseltamivir resistant strains occur and we re-validate the observation of Schauwvlieghe et al. (2018) in which severe influenza was identified as an independent risk factor for IPA-development in immunocompetent hosts.

Besides forming a good alternative antiviral drug, baloxavir marboxil, also has a different mechanism of action. As an influenza cap-dependent endonuclease inhibitor, baloxavir marboxil bypasses the possible negative effect related to the use of oseltamivir. Indeed, it is hypothesized that the neuraminidase inhibitor oseltamivir might inhibit the endogenous neuraminidase activity on the host immune cells although only limited inhibitory effects of oseltamivir on mammalian sialidases were reported $[15,16,34]$. Dewi et al. (2021) recently reported a detrimental effect of oseltamivir on fungal killing capacity of mouse splenocytes and human PBMCs in vitro [16]. Furthermore, in vivo, an increased susceptibility to IPA with higher fungal burden and mortality was found in oseltamivir treated mice [16]. However, although oseltamivir is suspected to have a potential adverse effect, our preclinical studies of oseltamivir and baloxavir marboxil, demonstrate the positive effect on influenza disease symptoms which counteracts the potential adverse effect on the fungal host response and the risk of acquiring IAPA [12].

This study highlights the importance of the timing of the baloxavir marboxil treatment. The earlier the treatment is started the better. However, in clinical practice, this is not easy to realize. The majority of the critically ill influenza patients in ICU have had influenza symptoms for more than a few days [35,36]. We now preclinically show that even if baloxavir marboxil treatment is delayed until 4 days post infection, we still observe marked improvements in our IAPA mice.

\section{Conclusions}

In conclusion, our results provide evidence that initiating early baloxavir marboxil can prevent a severe influenza infection thereby decreasing the susceptibility to a lethal Aspergillus co-infection. In the future, clinical studies can explore the therapeutic potential of baloxavir marboxil in fighting IAPA even when treatment-onset is postponed as early treatment is not always possible in clinical situation. In the broader context, we emphasize the importance to preclinically investigate potential pathogenic mechanisms and therapeutic interventions not only in IAPA but also in other VAPA's.

Author Contributions: Conceptualization, L.S., K.L., J.W. and G.V.V.; methodology, L.S., K.L., J.W. and G.V.V.; software, L.S., K.L., J.W. and G.V.V.; validation, L.S., K.L., J.W. and G.V.V.; formal analysis, L.S.; investigation, L.S., C.J., B.T., E.V. (Eliane Vanhoffelen), L.V.d.S. and E.V. (Erik Verbeken); resources, L.N., E.V. (Erik Verbeken), S.H.-B., K.L. and G.V.V.; data curation, L.S.; writing-original draft preparation, L.S.; writing-review and editing, L.S. and L.N.; E.V. (Erik Verbeken), S.H.-B., K.L., J.W. and G.V.V.; visualization, L.S.; supervision, G.V.V.; project administration, S.H.-B., K.L., J.W. and G.V.V.; funding acquisition, S.H.-B., K.L., J.W. and G.V.V. All authors have read and agreed to the published version of the manuscript.

Funding: This research was funded by the Research Foundation Flanders (FWO), project funding under Grants 1506114N, G053121N, G057721N] to G.V.V., J.W., S.H.-B. and K.L.; clinical research funding to J.W., Research Foundation Flanders (FWO), aspirant mandate under Grant $1186121 \mathrm{~N}$ to L.S.; KU Leuven internal funds] under Grant C24/17/061 to G.V.V.

Institutional Review Board Statement: The study was conducted according to the guidelines of the Declaration of Helsinki, and approved by the Institutional Review Board (or Ethics Committee) of KULeuven (P074/2018)).

Informed Consent Statement: Not applicable.

Data Availability Statement: Data available upon reasonable request to the corresponding author. 
Acknowledgments: The authors would like to thank Ria Van Berwaer for fine technical assistance and Anna Naumova for logistic support. All preclinical imaging was performed in the Molecular Small Animal Imaging Center (MoSAIC), a KU Leuven facility.

Conflicts of Interest: K.L. received consultancy fees from MRM Health, MSD and Gilead, speaker fees from FUJIFILM WAKO, Pfizer and Gilead and a service fee from Thermo fisher Scientific. J.W. has received investigator-initiated grants from Pfizer, Gilead and MSD and speakers' and travel fees from Pfizer, Gilead and MSD.

\section{References}

1. Fischer, J.J.; Walker, D.H. Invasive Pulmonary Aspergillosis Associated with Influenza. JAMA J. Am. Med. Assoc. 1979, 241, 1493-1494. [CrossRef]

2. Vanderbeke, L.; Spriet, I.; Breynaert, C.; Rijnders, B.J.A.; Verweij, P.E.; Wauters, J. Invasive pulmonary aspergillosis complicating severe influenza: Epidemiology, diagnosis and treatment. Curr. Opin. Infect. Dis. 2018, 31, 471-480. [CrossRef] [PubMed]

3. Verweij, P.E.; Rijnders, B.J.A.; Brüggemann, R.J.M.; Azoulay, E.; Bassetti, M.; Blot, S.; Calandra, T.; Clancy, C.J.; Cornely, O.A.; Chiller, T.; et al. Review of influenza-associated pulmonary aspergillosis in ICU patients and proposal for a case definition: An expert opinion. Intensive Care Med. 2020, 46, 1524-1535. [CrossRef]

4. Schauwvlieghe, A.F.A.D.; Rijnders, B.J.A.; Philips, N.; Verwijs, R.; Vanderbeke, L.; Van Tienen, C.; Lagrou, K.; Verweij, P.E.; Van De Veerdonk, F.L.; Gommers, D.; et al. Invasive aspergillosis in patients admitted to the intensive care unit with severe influenza: A retrospective cohort study. Lancet Respir. Med. 2018, 6, 782-792. [CrossRef]

5. Van de Veerdonk, F.L.; Kolwijck, E.; Lestrade, P.P.A.; Hodiamont, C.J.; Rijnders, B.A.; van Paassen, J.; Haas, P.-J.; dos Santos, C.O.; Kampinga, G.A.; Bergmans, D.C.J.J.; et al. Influenza-associated aspergillosis in critically ill patients. Am. J. Respir. Crit. Care Med. 2017, 196, 524-527. [CrossRef]

6. Wauters, J.; Baar, I.; Meersseman, P.; Meersseman, W.; Dams, K.; De Paep, R.; Lagrou, K.; Wilmer, A.; Jorens, P.; Hermans, G. Invasive pulmonary aspergillosis is a frequent complication of critically ill H1N1 patients: A retrospective study. Intensive Care Med. 2012, 38, 1761-1768. [CrossRef]

7. Liu, W.-L.; Yu, W.-L.; Chan, K.-S.; Yang, C.-C.; Wauters, J.; Verweij, P. Aspergillosis related to severe influenza: A worldwide phenomenon? Clin. Respir. J. 2019, 13, 540-542. [CrossRef]

8. Donnelly, J.P.; Chen, S.C.; Kauffman, C.A.; Steinbach, W.J.; Baddley, J.W.; Verweij, P.E.; Clancy, C.J.; Wingard, J.R.; Lockhart, S.R.; Groll, A.H.; et al. Revision and update of the consensus definitions of invasive fungal disease from the european organization for research and treatment of cancer and the mycoses study group education and research consortium. Clin. Infect. Dis. 2020, 71, 1367-1376. [CrossRef] [PubMed]

9. Feys, S.; Almyroudi, M.P.; Braspenning, R.; Lagrou, K.; Spriet, I.; Dimopoulos, G.; Wauters, J. A Visual and Comprehensive Review on COVID-19-Associated. J. Fungi 2021, 7, 1067. [CrossRef]

10. Segala, F.V.; Bavaro, D.F.; Di Gennaro, F.; Salvati, F.; Marrota, C.; Saracino, A.; Murri, R.; Fantoni, M. Impact of SARS-CoV-2 epidemic on antimicrobial resistance: A literature review. Viruses 2021, 13, 2110. [CrossRef]

11. Chen, L.; Han, X.; Li, Y.; Zhang, C.; Xing, X. Invasive pulmonary aspergillosis in immunocompetent patients hospitalised with influenza A-related pneumonia: A multicenter retrospective study. BMC Pulm. Med. 2020, 20, 239. [CrossRef] [PubMed]

12. Seldeslachts, L.; Vanderbeke, L.; Fremau, A.; Reséndiz-Sharpe, A.; Jacobs, C.; Laeveren, B.; Ostyn, T.; Naesens, L.; Brock, M.; Van De Veerdonk, F.L.; et al. Early oseltamivir reduces risk for influenza-associated aspergillosis in a double-hit murine model. Virulence 2021, 12, 2493-2508. [CrossRef] [PubMed]

13. Tobin, J.M.; Nickolich, K.L.; Ramanan, K.; Pilewski, M.J.; Lamens, K.D.; Alcorn, J.F.; Robinson, K.M. Influenza Suppresses Neutrophil Recruitment to the Lung and Exacerbates Secondary Invasive Pulmonary Aspergillosis. J. Immunol. 2020, 205, 480-488. [CrossRef]

14. Jaiswal, S.R.; Bhagwati, G.; Soni, M.; Thatai, A.; Aiyer, H.; Chakrabarti, S. Prophylactic oseltamivir during major seasonal influenza H1N1 outbreak might reduce both $\mathrm{H} 1 \mathrm{~N} 1$ and associated pulmonary aspergillosis in children undergoing haploidentical transplantation. Transpl. Infect. Dis. 2020, 22, 1-5. [CrossRef] [PubMed]

15. Hata, K.; Koseki, K.; Yamaguchi, K.; Moriya, S.; Suzuki, Y.; Yingsakmongkon, S.; Hirai, G.; Sodeoka, M.; von Itzstein, M.; Miyagi, T. Limited inhibitory effects of oseltamivir and zanamivir on human sialidases. Antimicrob. Agents Chemother. 2008, 52, 3484-3491. [CrossRef] [PubMed]

16. Dewi, I.M.W.; Cunha, C.; Jaeger, M.; Gresnigt, M.S.; Gkountzinopoulou, M.E.; Garishah, F.M.; Duarte-Oliveira, C.; Campos, C.F.; Vanderbeke, L.; Sharpe, A.R.; et al. Neuraminidase and SIGLEC15 modulate the host defense against pulmonary aspergillosis. Cell Rep. Med. 2021, 2, 100289. [CrossRef] [PubMed]

17. Ison, M.G.; Hayden, F.G.; Hay, A.J.; Gubareva, L.V.; Govorkova, E.A.; Takashita, E.; McKimm-Breschkin, J.L. Influenza polymerase inhibitor resistance: Assessment of the current state of the art-A report of the isirv Antiviral group. Antivir. Res. 2021, 194, 105158. [CrossRef]

18. Antiviral Drug Resistance among Influenza Viruses. 2016. Available online: https://www.cdc.gov/flu/professionals/antivirals/ antiviral-drug-resistance.htm (accessed on 21 August 2021). 
19. Behillil, S.; May, F.; Fourati, S.; Luyt, C.-E.; Chicheportiche, T.; Sonneville, R.; Tandjaoui-Lambiotte, Y.; Roux, D.; Guérin, L.; Mayaux, J.; et al. Oseltamivir resistance in severe influenza a (H1N1)pdm09 pneumonia and acute respiratory distress syndrome: A french multicenter observational cohort study. Clin. Infect. Dis. 2020, 71, 1089-1091. [CrossRef]

20. Hurt, A.C.; Hardie, K.; Wilson, N.J.; Deng, Y.-M.; Osbourn, M.; Leang, S.K.; Lee, R.T.C.; Iannello, P.; Gehrig, N.; Shaw, R.; et al. Characteristics of a widespread community cluster of H275Y oseltamivir-resistant a(H1N1)pdm09 influenza in Australia. J. Infect. Dis. 2012, 206, 148-157. [CrossRef]

21. Macesic, N.; Laplante, J.M.; Aaron, J.G.; DiMango, E.A.; Miko, B.A.; Pereira, M.R.; Reshef, R.; George, K.S. Baloxavir treatment of oseltamivir-resistant influenza A/H1pdm09 in two immunocompromised patients. Transpl. Infect. Dis. 2021, 23, 1-5. [CrossRef]

22. FDA Approves New Drug to Treat Influenza. 2018. Available online: https://www.fda.gov/news-events/press-announcements/ fda-approves-new-drug-treat-influenza (accessed on 21 August 2021).

23. Xofluza (Baloxavir Marboxil). 2021. Available online: https://www.ema.europa.eu/en/medicines/human/EPAR/xofluza (accessed on 11 August 2021).

24. Hayden, F.G.; Sugaya, N.; Hirotsu, N.; Lee, N.; De Jong, M.D.; Hurt, A.C.; Ishida, T.; Sekino, H.; Yamada, K.; Portsmouth, S.; et al. Baloxavir marboxil for uncomplicated influenza in adults and adolescents. N. Engl. J. Med. 2018, 379, 913-923. [CrossRef]

25. Kuo, Y.C.; Lai, C.C.; Wang, Y.H.; Chen, C.H.; Wang, C.Y. Clinical efficacy and safety of baloxavir marboxil in the treatment of influenza: A systematic review and meta-analysis of randomized controlled trials. J. Microbiol. Immunol. Infect. 2021, 54, 865-875. [CrossRef]

26. Fukao, K.; Ando, Y.; Noshi, T.; Kitano, M.; Noda, T.; Kawai, M.; Yoshida, R.; Sato, A.; Shishido, T.; Naito, A. Baloxavir marboxil, a novel cap-dependent endonuclease inhibitor potently suppresses influenza virus replication and represents therapeutic effects in both immunocompetent and immunocompromised mouse models. PLoS ONE 2019, 14, e0217307. [CrossRef]

27. Fukao, K.; Noshi, T.; Yamamoto, A.; Kitano, M.; Ando, Y.; Noda, T.; Baba, K.; Matsumoto, K.; Higuchi, N.; Ikeda, M.; et al. Combination treatment with the cap-dependent endonuclease inhibitor baloxavir marboxil and a neuraminidase inhibitor in a mouse model of influenza A virus infection. J. Antimicrob. Chemother. 2019, 74, 654-662. [CrossRef] [PubMed]

28. Resendiz-Sharpe, A.; Peres Da Silva, R.; Geib, E. Longitudinal multimodal imaging-compatible mouse model of triazole sensitive and resistant invasive pulmonary aspergillosis. Under Consideration.

29. Luther, K.; Rohde, M.; Sturm, K.; Kotz, A.; Heesemann, J.; Ebel, F. Characterisation of the phagocytic uptake of Aspergillus fumigatus conidia by macrophages. Microbes Infect. 2008, 10, 175-184. [CrossRef]

30. Chamilos, G. Aspergillus fumigatus and Aspergillosis in 2019. Am. Soc. Microbiol. Clin. Microbiol. Rev. 2020, 33, 1-75.

31. Nakamura, S.; Davis, K.M.; Weiser, J.N. Synergistic stimulation of type I interferons during influenza virus coinfection promotes Streptococcus pneumoniae colonization in mice. J. Clin. Investig. 2011, 121, 3657-3665. [CrossRef] [PubMed]

32. Salazar, F.; Bignell, E.; Brown, G.D.; Cook, P.C.; Warris, A. Pathogenesis of Respiratory Viral and Fungal Coinfections. Clin. Microbiol. Rev. 2021. [CrossRef]

33. Sun, K.; Metzger, D.W. Inhibition of pulmonary antibacterial defense by interferon- $\gamma$ during recovery from influenza infection. Nat. Med. 2008, 14, 558-564. [CrossRef]

34. Bigot, P.; Auffret, M.; Gautier, S.; Weinborn, M.; Ettahar, N.K.; Coupé, P. Unexpected platelets elevation in a patient with idiopathic thrombocytopenia treated with oseltamivir for influenza infection. Fundam. Clin. Pharmacol. 2016, 30, 483-485. [CrossRef]

35. Groeneveld, G.H.; Marbus, S.D.; Ismail, N.; de Vries, J.J.; Schneeberger, P.; Oosterheert, J.J.; van Dissel, J.T.; de Boer, M.G. Effectiveness of oseltamivir in reduction of complications and 30-day mortality in severe seasonal influenza infection. Int. J. Antimicrob. Agents 2020, 56, 106155. [CrossRef] [PubMed]

36. Muthuri, S.G.; Venkatesan, S.; Myles, P.; Leonardi-Bee, J.; Al Khuwaitir, T.S.A.; Al Mamun, A.; Anovadiya, A.; Azziz-Baumgartner E.; Báez, C.; Bassetti, M.; et al. Effectiveness of neuraminidase inhibitors in reducing mortality in patients admitted to hospital with influenza A H1N1pdm09 virus infection: A meta-analysis of individual participant data. Lancet Respir. Med. 2014, 2, 395-404. [CrossRef] 